\title{
Understanding failures in getting it up: The prevalence and predictors of failed ureteral access in ureteroscopy
}

Callum A. Lavoie; Max Levine; Trevor D. Schuler; Timothy A. Wollin; Shubha De Division of Urology, Department of Surgery, University of Alberta, Edmonton, AB, Canada

Cite as: Lavoie CA, Levine M, Schuler TD, et al. Understanding failures in getting it up: The prevalence and predictors of failed ureteral access in ureteroscopy. Can Urol Assoc J 2020 August 7; Epub ahead of print. http://dx.doi.org/10.5489/cuaj.6059

Published online August 7, 2020

$* * *$

\section{Abstract}

Introduction: Failed access ureteroscopy (FA) describes the inability to gain adequate access to a stone to allow for treatment. The purpose of this study was to identify the prevalence of, and factors predicting FA in patients presenting with renal and ureteral stones.

Methods: We performed a retrospective review of all ureteroscopy procedures performed for renal and ureteral stones by three endourologists over a six-month period at our center. All patients who underwent ureteroscopy (URS) for the purpose of stone treatment were included. Patients were excluded if they underwent URS for non-stone diagnosis or treatment. FA was investigated in relation to demographics, medical history, stone-specific characteristics, procedure-specific characteristics, etc. Statistical analysis consisted of descriptive statistics, as well as chi-squared and t-test analysis using SPSS statistical software version 24.0.

Results: A total of 188 cases were reviewed, with $8 \%$ of patients experiencing FA. Patient age, gender, body mass index (BMI), American Society of Anesthesiologists (ASA) score, emergency cases, previous stone treatment, use of computed tomography (CT) imaging, presence of hydronephrosis, and surgeon did not differ significantly between FA and successful access (SA) groups. Stone size $(9.88 \pm 5.8$ vs. $8.76 \pm 4.3 \mathrm{~mm}$; $\mathrm{p}=0.361)$ was also not significantly different. However, a significant difference was noted in time from first diagnosis to ureteroscopy (128 vs. 65 days; $\mathrm{p}=0.044$ ) between the FA and SA groups. Similarly, for ureteral stones, the FA group had a significantly greater proportion of stones located in the proximal ureter $(62.5 \%$ vs. $22.0 \%$; $\mathrm{p}=0.043$ ).

Conclusions: Proximal ureteric stones were more likely to result in FA ureteroscopy, and FA procedures were more likely to be preceded by extended time from first diagnosis to URS. Further investigation is necessary, and all endourology centers should track their own personal 
outcome data to allow for more meaningful analysis to be performed to improve patient outcomes.

\section{Introduction}

Ureteroscopy (URS) is considered a first line treatment option for the management of nephrolithiasis $<2 \mathrm{~cm}$ in maximal diameter. ${ }^{1}$ Risks specific to endoscopic management of stones include ureteric injury and the possibility of failed access. Failed access (FA) during URS denotes the inability to advance a ureteroscope to the level of the stone of interest, thereby preventing intervention. In the face of FA, an indwelling ureteric stent is typically left in place to temporize the ureteric obstruction and to facilitate subsequent access to the ureter via passive dilation with a repeat operative intervention ultimately being required for definitive stone management. ${ }^{1}$

Patients should be quoted a rate of FA as it pertains to their pre-operative risk discussion. The frequency of FA in the literature varies from 7.7-16\%. ${ }^{2-5}$ The risk of FA is hypothesized to be related to both patient and stone specific factors. A recent study has suggested that prior stone surgery and prior ipsilateral stent placement were protective against FA and the requirement for primary stenting 5 . A recent report from Fuller et al (2016) suggests that proximal stone location and younger females are at highest risk for FA during primary URS. ${ }^{3}$

The purpose of the present study seeks to examine the rates of FA at our institution amongst our endourology group. We hypothesized that FA rate would be $10 \%$ from anecdotal accounts.

\section{Methods}

After receiving institutional ethics approval, a retrospective analysis of all ureteroscopy procedures performed over a six-month period, by our institution's endourologists was undertaken, comparing patients with FA and successful access (SA). We excluded cases of retreatment via URS, patients with pre-operative ureteral stent placement, and diagnostic or therapeutic URS done for non-stone pathology.

Surgical technique was at the discretion of the surgeon. Ureteroscopy (semi-rigid $8 F r$ Slimline Gyrus ACMI and flexible URF-V, Olympus) included routine use of safety wires, and ureteral access sheaths. Difficult ureteric access was approached with any combination of dilation techniques (i.e. $8 / 10 \mathrm{Fr}$ coaxial dilation; $12 \mathrm{Fr}$ access sheath trocar dilation (followed by $14 \mathrm{Fr}$ sheath placement), $18 \mathrm{Fr}$ balloon dilation (in select circumstances), or back loading/railroad over a working-wire \pm alongside a safety wire.

Collected data included patient age, gender, BMI, ASA score, prior surgical/urologic/medical history, stone characteristics, and ureteric access status. Patient charts 
as well as diagnostic imaging and operative reports were reviewed for data abstraction through the electronic medical record. Demographic patient data, stone characteristic, and pre-operative factors are reported as mean ( \pm standard deviation) or as binary outcomes (yes/no) where appropriate for those who experienced FA vs. SA. FA was defined as the inability to advance a ureteroscope (semi-rigid or flexible) to an adequate point in the ureter to allow treatment of the ureteric or renal stone of interest (not including aberrant or complex intrarenal anatomy) requiring the procedure to be aborted. Two groups (FA and SA) were defined and compared with respect to the prevalence of pre-operative/stone factors. Prevalence in each group were compared using the Chi-square test or unpaired, two-tailed t-test, where appropriate, with an $\alpha=$ 0.05 . Odds ratios for experiencing FA with $95 \%$ confidence intervals were calculated to estimate risk.

\section{Results}

A total of 245 ureteroscopies were reviewed, 188 of which met inclusion criteria and were analyzed. The FA rate was $8 \%(15 / 188)$ and the SA rate was $92 \%(173 / 188)$. The gender distribution was well balanced in the two groups ( $53 \%$ male SA vs $60 \%$ male FA, $\mathrm{p}=0.583$ ). Average age was not significantly different between those with SA and FA (50.9 vs. 57.7 years; $\mathrm{p}=0.067$ ), nor was there any difference between genders. The average BMI did not significantly differ between the groups ( 30.0 vs. $31.5 ; \mathrm{p}=0.41)$, and there was no difference in mean pre-op ASA score (1.96 vs. $2.13 ; p=0.277)$. Approximately half of all patients had prior interventions for previous stone disease (SA 46\%, FA 50\%), with no significant differences in prior rates of ureteroscopy (SA 16.2\%, FA 31.3\%), shockwave lithotripsy (SA 16\%, FA 32\%), or percutaneous nephrolithotomy (SA 7.4\%, FA 6.3\%).

Stone characteristics were also relatively similar between the SA and FA groups. Average stone size was not significantly different between the SA and FA groups $(8.8 \mathrm{~mm}$ vs. $9.9 \mathrm{~mm} ; \mathrm{p}=0.361$ ). The distribution of stones residing in the distal, mid, and proximal ureter, ureteropelvic junction (UPJ), or intra-renally did not significantly differ between the groups on Chi-square analysis. Distal stones had a FA rate of $4.5 \%$, while proximal ureteric stones had a FA rate of $18.5 \%$ (Figure 1). Proximal ureteric stones carried a significantly higher risk of FA $(\mathrm{OR}=4.773,95 \% \mathrm{CI}$ : $1.053-21.635$ (Figure 1). When FA rate was examined according to surgeon, the rates of FA did not reach significance $(11.8 \%$ vs $1.7 \%$ vs. $9.3 \% ; p=0.152)$.

\section{Discussion}

Failed access is a potential risk to undergoing URS that requires placement of a stent and a return to OR at a later date for a repeat procedure. The risk of FA should be clearly discussed with patients as part of their informed consent discussion. ${ }^{1}$ Accuracy in quoted risk improves the quality of consent discussion, as rates of FA vary in the literature from $7.7-16 \% .^{2-5}$

$\mathrm{Ji}$ and colleagues 2012 found a FA rate of $11.5 \%$ in a prospective study designed to assess the outcomes of second attempt URS when double-J stenting vs ureteral catheter 
placement below the level of impacted stone. ${ }^{4}$ This group utilized rigid URS only. They approached difficult ureters with the sole use of balloon dilation and passage of double-J stent or ureteral catheter for ongoing inability to advance the ureteroscope. In a retrospective review of 120 consecutive ureteroscopies by a single surgeon, Viers et al (2015) quantified a FA rate of $16 \%$. The surgeon analyzed attempted insertion of ureteral access sheaths routinely for flexible URS cases, with difficult passage managed with dilation using the sheath trocar, downsized access sheath, coaxial dilation, balloon dilation, or passage of the scope over a wire. ${ }^{5}$ The management of difficult ureters with semi-rigid URS was not defined. A similarly designed study by Shields et al (2009) revealed a FA rate of $13.1 \%{ }^{6}$ Fuller and colleagues (2016) in a multi-institutional retrospective review of unstented patients calculated an overall FA rate of $7.7 \%{ }^{3}$ The approaches to the difficult ureter varied between the three institutions, with one using routine balloon dilation, the second using coaxial dilation, and the third not using any dilation when difficulty was encountered. The rates of FA varied between the institutions $(22.9 \%, 4.5 \%$, and $4.2 \%)$, with the higher failure rates being accounted for by a surgeon in early practice, and is likely reflective of an expected, more conservative operative approach.

In the current study, our local data demonstrates a FA rate of $8 \%$ amongst three of our endourology subspecialists. The rates of FA may vary according to which surgeon is analyzed, however with our sample size the difference between surgeons did not reach statistical significance $(11.8 \%$ vs $1.7 \%$ vs. $9.3 \% ; p=0.152)$. All three surgeons in the analysis were well beyond their learning curve for ureteroscopy with the length of practice varying between 10 and 20 years. The approach to difficult ureters was not standardized between surgeons, as each surgeon followed their personal practice. Despite showing no statistical difference between surgeons' rates of FA, these numbers reflect each surgeon's own personal rates of FA, and thus provide them with real life numbers to convey to their patients in their risk counseling at our centre.

Though the rates of failed access appear to vary between urologists included in this analysis, limited conclusions can be objectively drawn. Given this is a pooled patient population the surgeons were exposed to similar patients during the studied dates. This could be an effect of our relatively small sample sizes, but technical aspects to troubleshooting should also be considered. We reviewed the decision tree for difficult to access ureters with the surgeons included in this analysis, and no significant differences in technical approach were identified aside from one surgeon infrequently utilizing balloon dilation in selective scenarios.

While this does not account for subtle differences in technique or applied force, there were also no differences ascertained upon review of patients' operative reports. Additionally, it is important to acknowledge that although the surgeons did not communicate any differences in their operative decision-making algorithms, there will undoubtedly be some variation on a caseby-case basis, and each surgeon will exhibit different thresholds for continued attempts to gain access versus electing to place a stent and returning to the OR at a later date for a second attempt. 
Aside from the possible influence of the operating surgeon determining risk of FA, our data finds few pre-operative stone or patient factors to reliably predict a greater or worse FA rate. We found gender, stone size as a continuous or discrete variable $<1 \mathrm{~cm} \mathrm{vs}>1 \mathrm{~cm}$ ), and prior stone surgery (ESWL, ureteroscopy, percutaneous nephrolithotomy) did not significantly impacted the risk of FA. We did however find that having a stone in the proximal ureter significantly increased the risk of FA (OR 4.77, 95\% CI: 1.05-21.6).

The association of FA with proximal ureteral stones was also reported by Fuller et al (2016). They found proximal ureteric stones had the highest failure rates (18.3\%) and on multivariable logistic regression proximal ureteric stones were a significant predictor of FA $(\mathrm{OR}=3.14)$. Their analysis found that overall, stones $>10 \mathrm{~mm}$ were associated with lower FA rates, but this was determined to likely be an artifact of large intrarenal stones as there was no relationship between stone size either absolutely or categorically (with a $10 \mathrm{~mm}$ cut-off point) when ureteric stones were analyzed separately. Our study population also observed the highest failure rate in stones found in the proximal ureter (18.5\%) and that stone size was not predictive of FA. In addition, we found that proximal ureteric stones confer an odds ratio of 4.77 for the occurrence of FA, similar to that reported by Fuller and colleagues. The congruence of these risk values suggests the true increased risk of FA for proximal ureteric stones is approximately 3-5 times the baseline risk of FA. Reasons for this may include the relatively greater mobility of the proximal ureter reducing purchase on attempts at dilation/scope advancement. Additionally, stones in the proximal ureter may be signify a uniformly narrow ureter throughout its entire course. Surgeons may also be less aggressive in the proximal ureter, feeling the risk of complications may be higher.

Technical issues which could have impacted our failed access rate may include the use of 8.5Fr flexible ureteroscopes with $12 / 14$ access sheaths, which as compared to slimmer ureteroscopes, may have limited our ability to traverse moderately narrowed ureters. Also, at our center patients are typically anesthetized without paralysis, which may play a role in muscle relaxation and ultimately lead to more difficult access to the upper GU tract.

This analysis is limited by its retrospective design and sample size. We chose to examine the FA rates of 3 endourologists and did not include those surgeons whose practice is subspecialized in other urological disciplines. This potentially limits generalizability of our results to others performing URS. Furthermore, the approach to difficult ureteric access may have varied slightly from surgeon to surgeon, as a protocol was not standardized and each surgeon operated according to their own practice pattern. The variability in access to different scope models/sizes at different centres may also impact the true FA risk.

\section{Conclusions}

conclusion, we found our FA rate across 3 experienced endourologists was $8 \%$, increasing to $18.5 \%$ when stones were in the proximal ureter $(\mathrm{OR}=4.77)$. The data from this study provides 
refined risk values to aid in pre-operative counseling and makes a case for all surgeons to track personal outcome data in order to quantify their personal rates of FA that can aid in patient counseling and quality improvement. 


\section{References}

1. Ordon M, Andonian S, Blew B, Schuler T, Chew B, Pace KT. CUA guideline: Management of ureteral calculi. Can Urol Assoc J. 2015;9(11-12):E837-51.

2. Cetti RJ, Biers S, Keoghane SR. The difficult ureter: What is the incidence of prestenting? Ann R Coll Surg Engl. 2011;93(1):31-33.

3. Fuller T, Rycyna KJ, Ayyash OM, et al. Defining the rate of primary ureteroscopic failure in unstented patients: A multi-institutional study. J Endourol. 2016.

4. Ji C, Gan W, Guo H, et al. A prospective trial on ureteral stenting combined with secondary ureteroscopy after an initial failed procedure. Urol Res. 2012;40(5):593-598.

5. Viers BR, Viers LD, Hull NC, et al. The difficult ureter: Clinical and radiographic characteristics associated with upper urinary tract access at the time of ureteroscopic stone treatment. Urology. 2015.

6. Shields JM, Bird VG, Graves R, Gomez-Marin O. Impact of preoperative ureteral stenting on outcome of ureteroscopic treatment for urinary lithiasis. J Urol. 2009;182(6):2768-2774.

7. Fabrizio MD, Behari A, Bagley DH. Ureteroscopic management of intrarenal calculi. $J$ Urol. 1998;159(4):1139-1143.

8. Hollenbeck BK, Schuster TG, Faerber GJ, Wolf JS,Jr. Comparison of outcomes of ureteroscopy for ureteral calculi located above and below the pelvic brim. Urology. 2001;58(3):351-356.

9. Perlmutter AE, Talug C, Tarry WF, Zaslau S, Mohseni H, Kandzari SJ. Impact of stone location on success rates of endoscopic lithotripsy for nephrolithiasis. Urology. 2008;71(2):214-217.

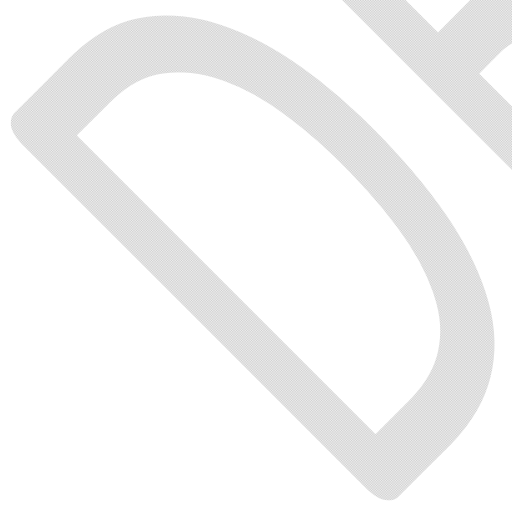




\section{Figures and Tables}

Fig. 1. Failed access rate by stone location.

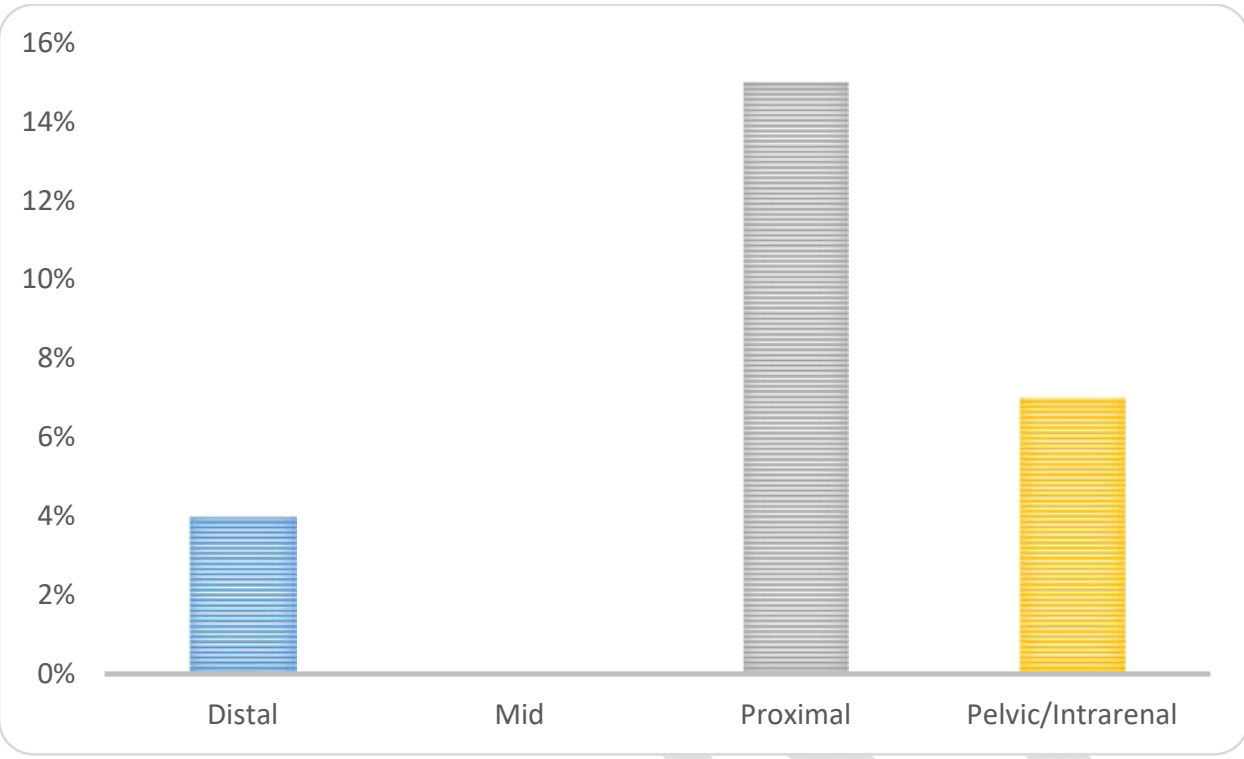

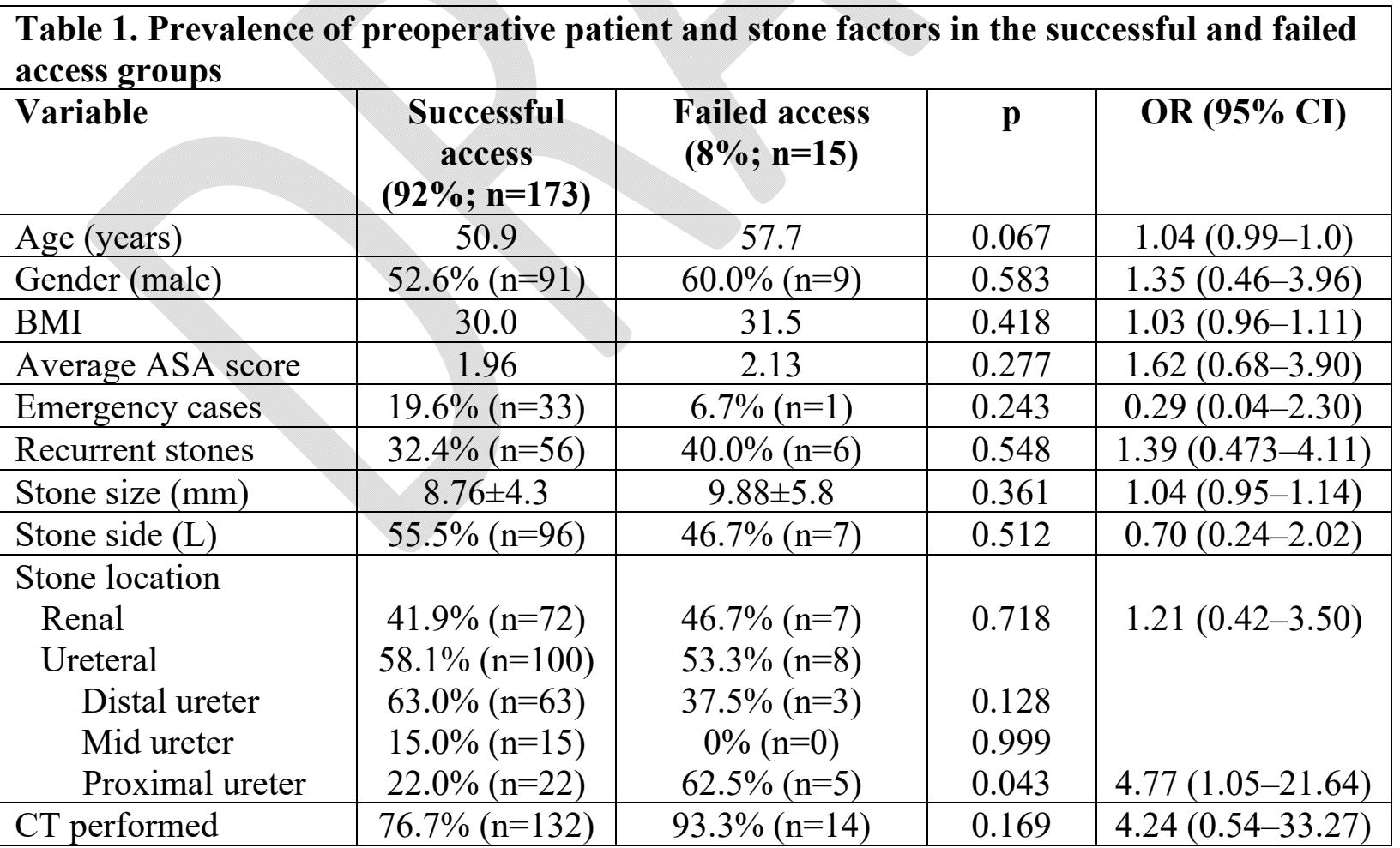




\begin{tabular}{|l|c|c|c|c|}
\hline Hydronephrosis & $45.7 \%(\mathrm{n}=79)$ & $60.0 \%(\mathrm{n}=9)$ & 0.372 & $1.63(0.56-4.79)$ \\
\hline $\begin{array}{l}\text { Time from first } \\
\text { diagnosis to URS }\end{array}$ & 65 days & 128 days & 0.044 & $1.00(1.00-1.01)$ \\
\hline Surgeon & $43.4 \%(\mathrm{n}=75)$ & $66.7 \%(\mathrm{n}=10)$ & 0.152 & $($ ref $)$ \\
A & $34.1 \%(\mathrm{n}=59)$ & $6.7 \%(\mathrm{n}=1)$ & 0.052 & $0.13(0.02-1.02)$ \\
B & $22.5 \%(\mathrm{n}=39)$ & $26.7 \%(\mathrm{n}=4)$ & 0.674 & $0.77(0.23-2.61)$ \\
\hline
\end{tabular}

ASA: American Society of Anesthesiologists; BMI: body mass index; CI: confidence interval; CT: computed tomography; OR: odds ratio; URS: ureteroscopy. 


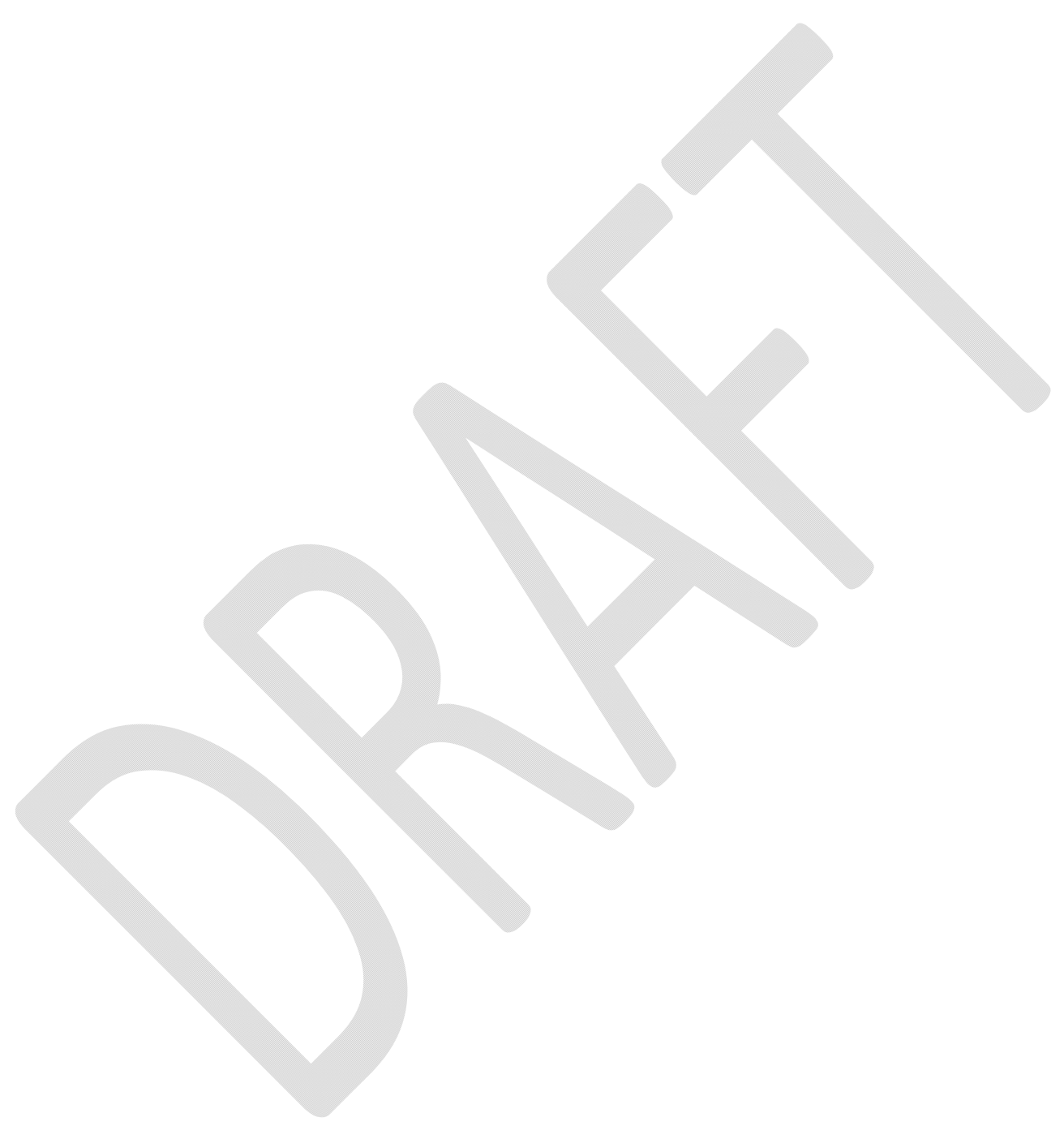




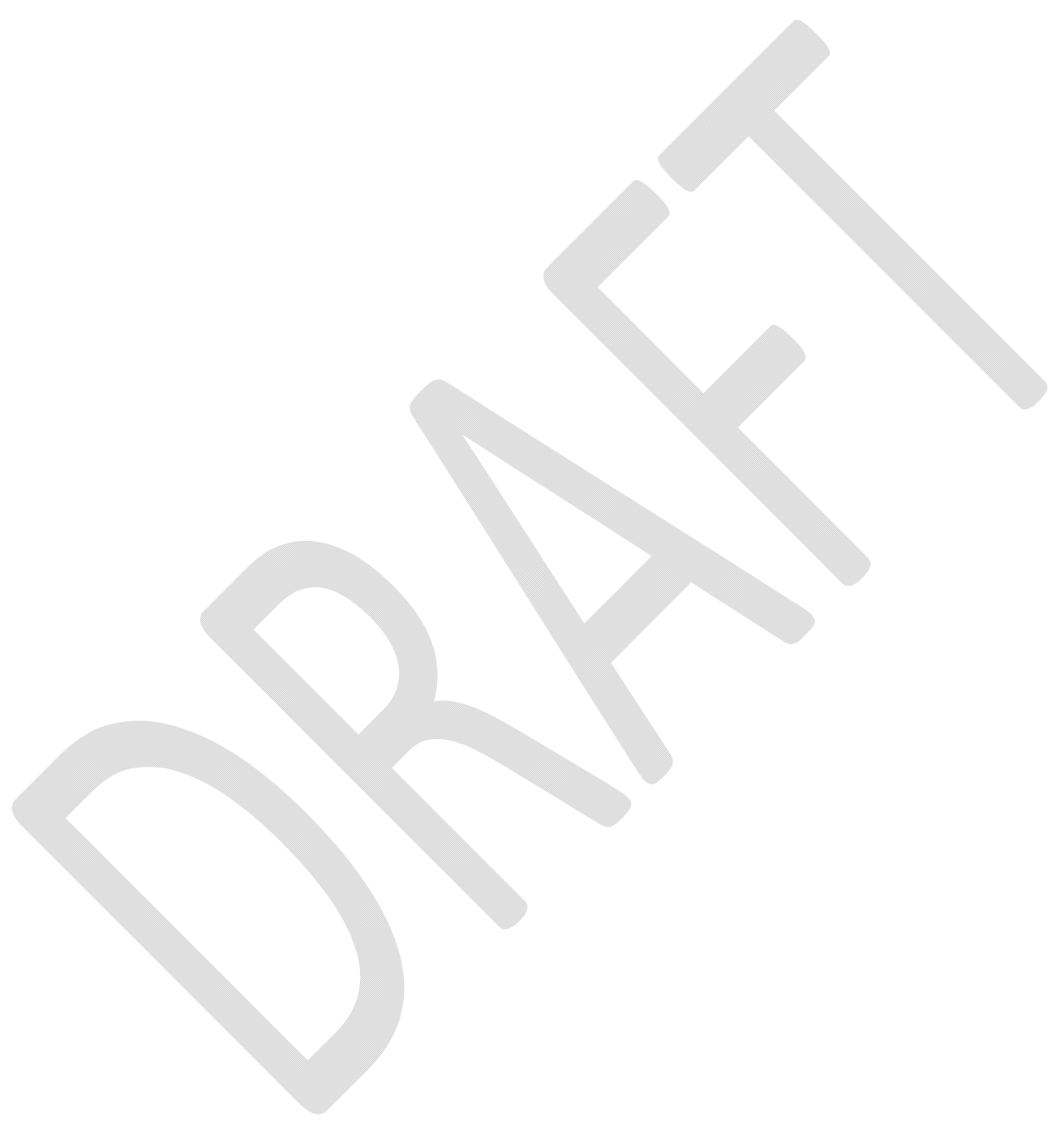

